\title{
Tumor de Frantz : Presentación de un caso clínico.
}

Tumor de Frantz: Presentation of a clinical case.

Carlos Xavier Haro Erazo ${ }^{1} \&$ Felipe Alfonso Salinas Mancheno ${ }^{2}$

Recibido: 28-04-2019/ Revisado: 25-05-2019/Aceptado: 24-06-2019/ Publicado: 05-07-2019

\begin{abstract}
.
DOI: https://doi.org/10.33262/cienciadigital.v3i3.664

Pseudopapillary tumor of pancreas lesion benign in $90 \%$ and malignant in $10 \%$, this neoplasm is defined as not worked little is known as unknown etiology is not postulated a hormonal origin is found in its relationship with the most common presentation groups women in Reproductive stage, $85 \%$ of cases is reported as a lesion involving only the pancreas, and $15 \%$ as a lesion or generalized carcinomatosis or metastases in spleen liver peritoneal colon and lymph nodes. We present the case of a 16-year-old patient with abdominal pain in the epigastrium, a feeling of fullness, who were like gastritis, in the physical examination presents pain in the epigastrium with a biochemical profile, tumor markers within normal TAC, dependent occupational injury of pancreas
\end{abstract}

Key Words: Frantz, Pancreas, Neoplasia Beninga, Women In Fertil Age

\section{Resumen.}

Tumor seudopapilar de páncreas corresponde de 1 a $2 \%$ de todas las neoplasias del páncreas se la define como no funcionante, poco frecuente de etiología desconocida sin embargo se postula un origen hormonal de ahí su relación con los grupos de presentación más común mujeres en etapa reproductiva, siendo más frecuente en mujeres de hasta la tercera década de vida, quienes con una resección quirúrgica completa, tienen una sobrevida del $90 \%$ a cinco años. Se considera de comportamiento benigno debido a su bajo poder metastático (alrededor del 15\%), siendo infrecuente la afección ganglionar. Debido a su crecimiento insidioso sin mayor sintomatología, sin cambios metabólicos, ni elevación de biomarcadores, el diagnóstico de esta patología es difícil $(2,12)$ Presentamos el caso de una paciente femenina de 16 años con dolor abdominal en epigastrio, sensación de llenura, que fueron tratados como una gastritis, al examen físico presenta dolor leve en epigastrio cuenta con perfil bioquímico, marcadores tumorales dentro de la normalidad a la

\footnotetext{
${ }^{1}$ Universidad Central del Ecuador. Médico General. carlox.chex87@hotmail.com

${ }^{2}$ Escuela Superior Politécnica de Chimborazo, Médico General. felipesalinas151@yahoo.com
} 
TAC lesión ocupativa dependiente de páncreas.

Palabras Clave: Frantz, Pancreas , Neoplasia Benigna, Mujer Edad Fertil.

\section{Introducción.}

El tumor pseudopapilar sólido (SPT) del páncreas, reportado por primera vez por Frantz et al1 en 1959, es una neoplasia pancreática poco común pero distinta, que representa el $1 \%$ $2 \%$ de todos los tumores pancreáticos. El tumor recibió varios nombres diferentes de acuerdo con su carácter macroscópico y microscópico hasta que este nombre, tumor pseudopapilar sólido del páncreas, fue definido por la Organización Mundial de la Salud (OMS) como un tumor único en 1996(1,8)

El tumor es nueve veces más frecuente en el cuerpo que en la cola del páncreas. La mayoría de los pacientes son mujeres jóvenes y la mayoría de ellos son asintomáticos, las dificultades con el diagnóstico preoperatorio, junto con la alta incidencia de tumores con posible malignidad y el buen resultado con la resección, sugieren que todos los tumores quísticos sospechosos del páncreas deban resecarse.(4)

Existen estudios retrospectivos uno de los más interesantes de 13 años publicado en American Roentgen Ray Society en el cual se captan 19 pacientes con tumor seudopapilar de páncreas los mismos que presentaron clínica de dolor en epigastrio crónico, difuso sensación de plenitud temprana, la mediana de tiempo de intervalos entre los síntomas y diagnostico 5 meses en un rango de ( 0 días a 4 años), los resultados de laboratorio de exámenes hepatobiliares y pancreáticos fueron normales en 16 de los 19 pacientes y 3 presentaban alteraciones moderadas en la bilirrubina lipasa y amilasa, 18 de los 19 casos se resolvieron por resección del tumor ya sea por técnicas de resección de whipple o duodenectomia pancreática distal temprana, 1 solo caso debido a que involucraba vasos mesentéricos e intestino delgado fue irresecable.(6)

No obstante, el tratamiento quirúrgico, presenta un excelente pronóstico tras resección completa incluso si existen metástasis. (10)

\section{Caso Clínico.}

Paciente femenina de 16 años de edad con antecedentes patológicos de gastritis crónica sin tratamiento con diagnostico endoscópico de un año de evolución, acude a servicio de emergencia en clínica particular por presentar cuadro clínico de dolor abdominal con irradiación lumbar escala de EVA 4/10 acompañada de sensación de plenitud ocasionalmente por lo que prescriben medicación que no especifica recibe alivio temporal pero molestias vuelven, por cuadros recurrentes acude a SOLCA CHIMBORAZO donde luego de valoración clínica y hallazgos clínicos se solicita exámenes complementarios.

Endoscopia Digestiva Alta: Estómago: sobre el tercio proximal de la unión de la pared posterior y la curvatura mayor, compresión externa dura al contacto con la pinza de biopsia. Histopatológico mucosa gástrica - gastritis crónica activa leve no atrófica - Helicobacter 
pylori $(++)$ - grupo II.

Gráfico No1. Endoscopia Digestiva Alta
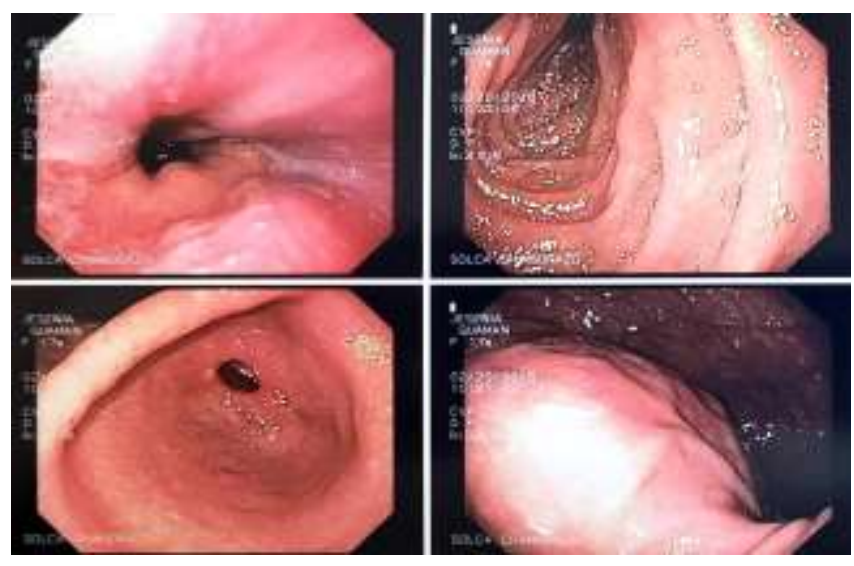

Fuente: Eda servicio de gastroenterologia solca chimborazo 2018

Tomografía simple y contrastada tórax abdomen y pelvis: Hígado de morfología, tamaño y localización conservada. En el segmento VIII se aprecia una imagen redondeada hipodensa, definida, con densidad de líquido en la fase simple y contrastada que mide $37 \mathrm{~mm}$; se aprecia otra lesión de similares características en el segmento VII que mide $13 \mathrm{~mm}$. Se aprecia una lesión ocupativa retroperitoneal dependiente del cuerpo y cola del páncreas, la misma que comprime parcialmente las asas intestinales adyacentes, es heterogénea con contenido de predominio hipodenso, e isodenso periférico, mide aproximadamente $118 \mathrm{x}$ 109 x $94 \mathrm{~mm}$, con un volumen aproximado de 628cc. La cabeza del páncreas impresiona respetada.

Gráfico No2 Tomografía simple y contrastada tórax abdomen y pelvis:

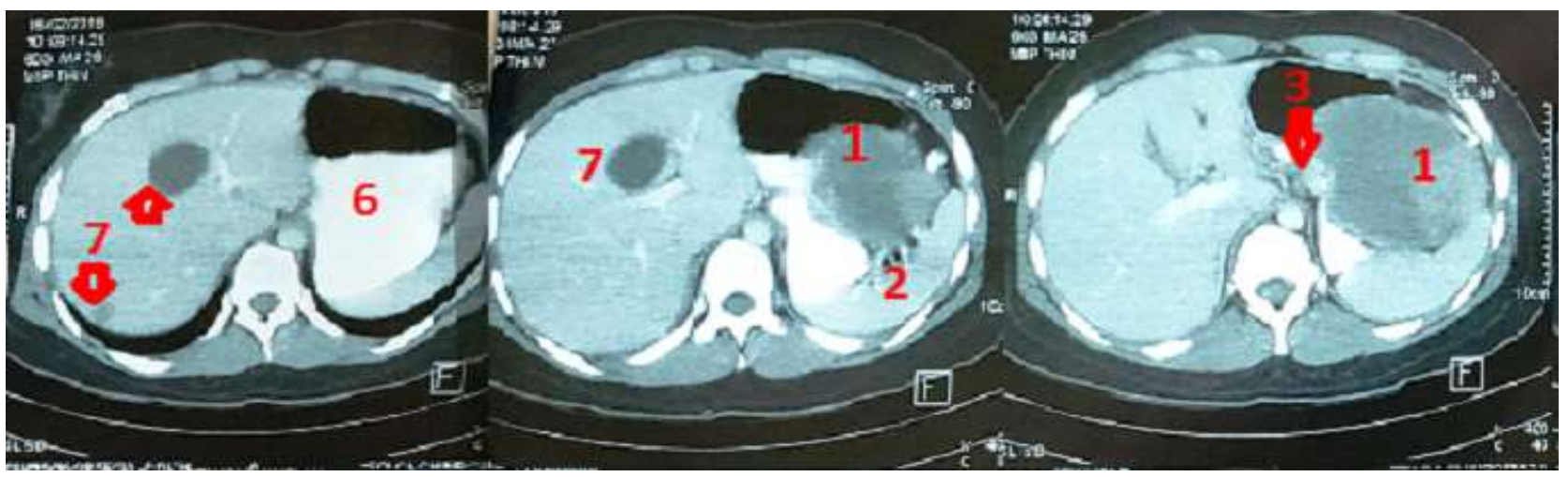




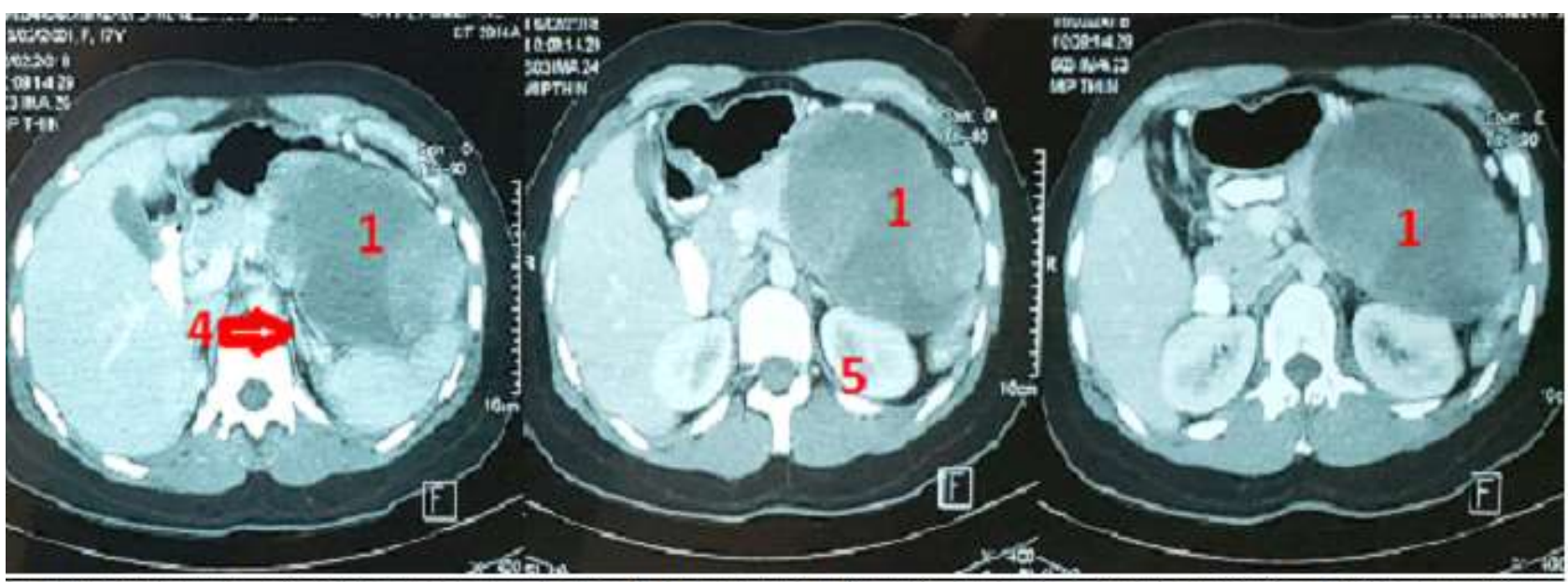

1) Lesión tumoral de páncreas 2) Bazo 3) Páncreas 4) Hilio Esplénico 5) Riñón izquierdo 6) Cavidad gástrica 7) Qui stes Hepáticos.

Fuente: Tac contrastada servicio imagenologia solca Chimborazo 2018

Resonancia Magnética: Páncreas se observa masa dependiente de cuerpo y cola es de contornos definidos tiene capsula, está desplazando hacia adelante al estómago es de intensidad mixta, los diámetros de la lesión son 118x84x127mm volumen $679 \mathrm{cc}$.

Gráfico No3 Resonancia Magnética

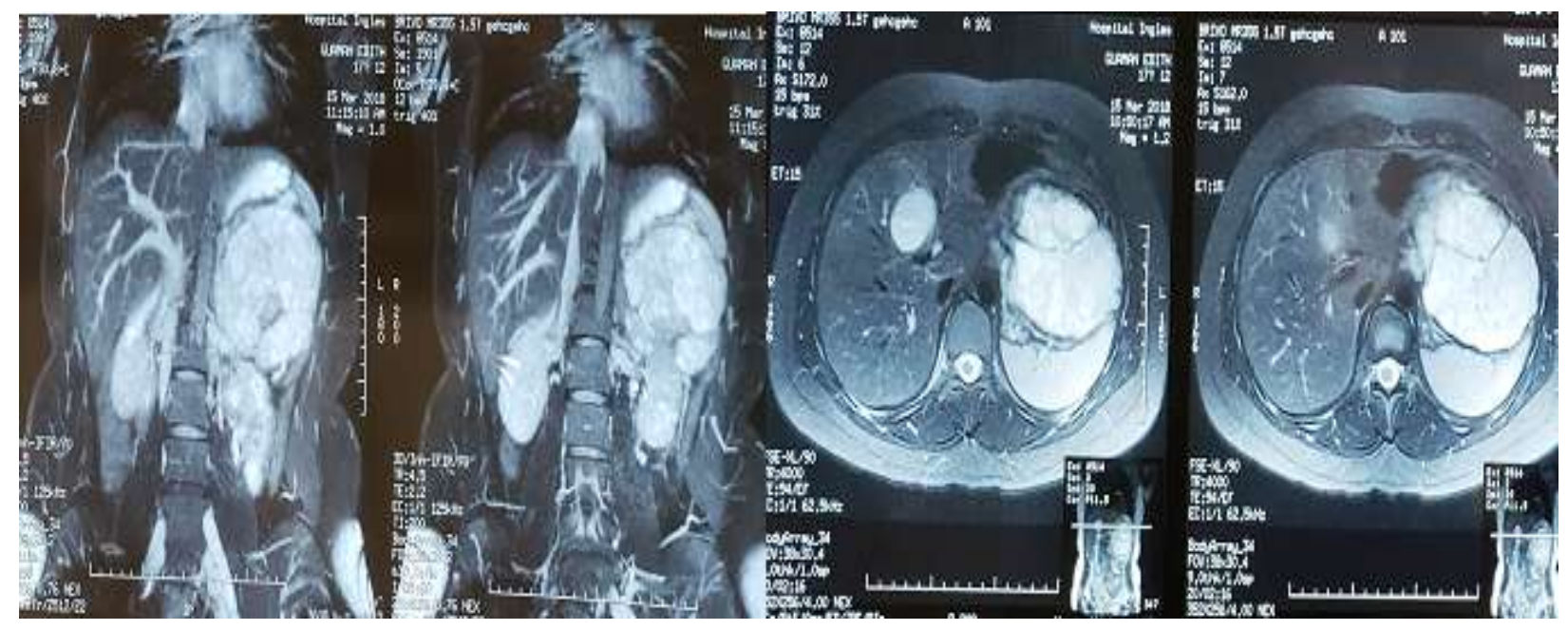

Fuente: Rnm Servicio Imagenologia Solca Chimborazo 2018

Procedimiento Quirúrgico: De forma electiva, encontrando masa tumoral dependiente de cuerpo y cola de páncreas de 120x $150 \mathrm{~mm}$ de contenido mixto sólido y quístico firmemente adherida a la arteria y vena esplénica por lo cual se realiza exéresis en bloque de cuerpo y cola de páncreas afectados por masa tumoral más esplenectomía 
Gráfico No4 Procedimiento Quirúrgico

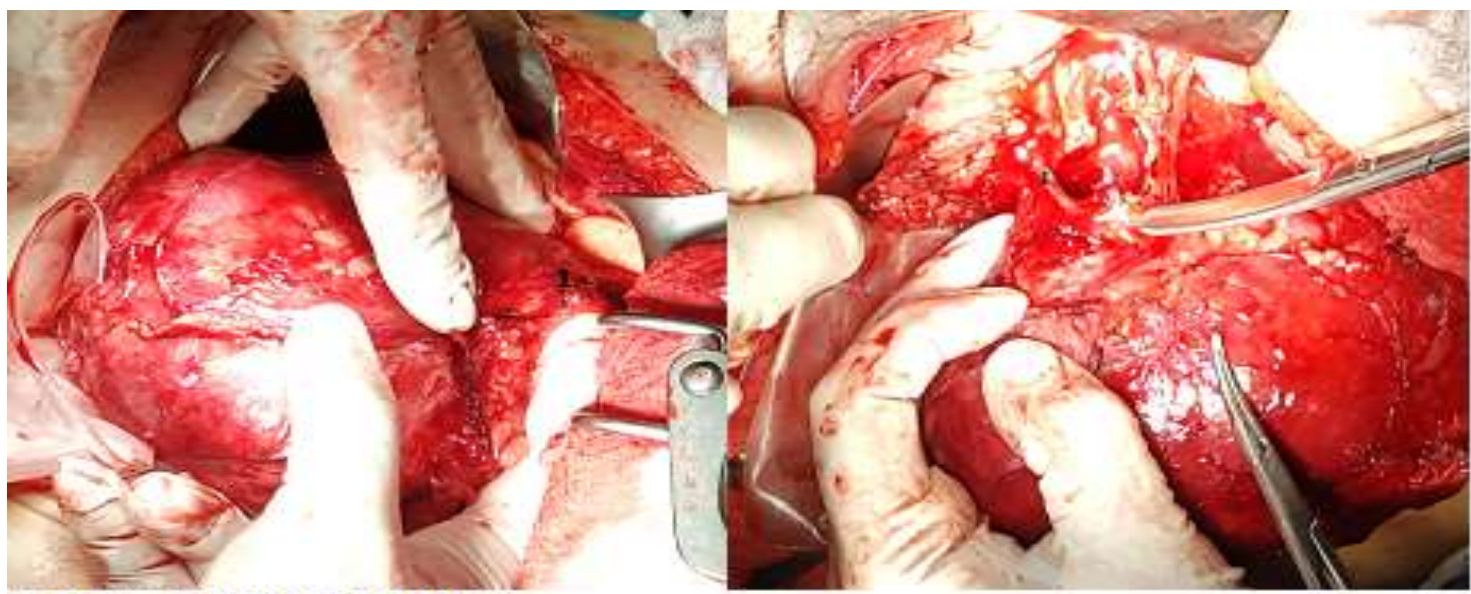

1. Vena y Arteria Esplénica

FUENTE: PROCECIDIMENTO QUIRURGICO SOLCA CHIMBORAZO 2018

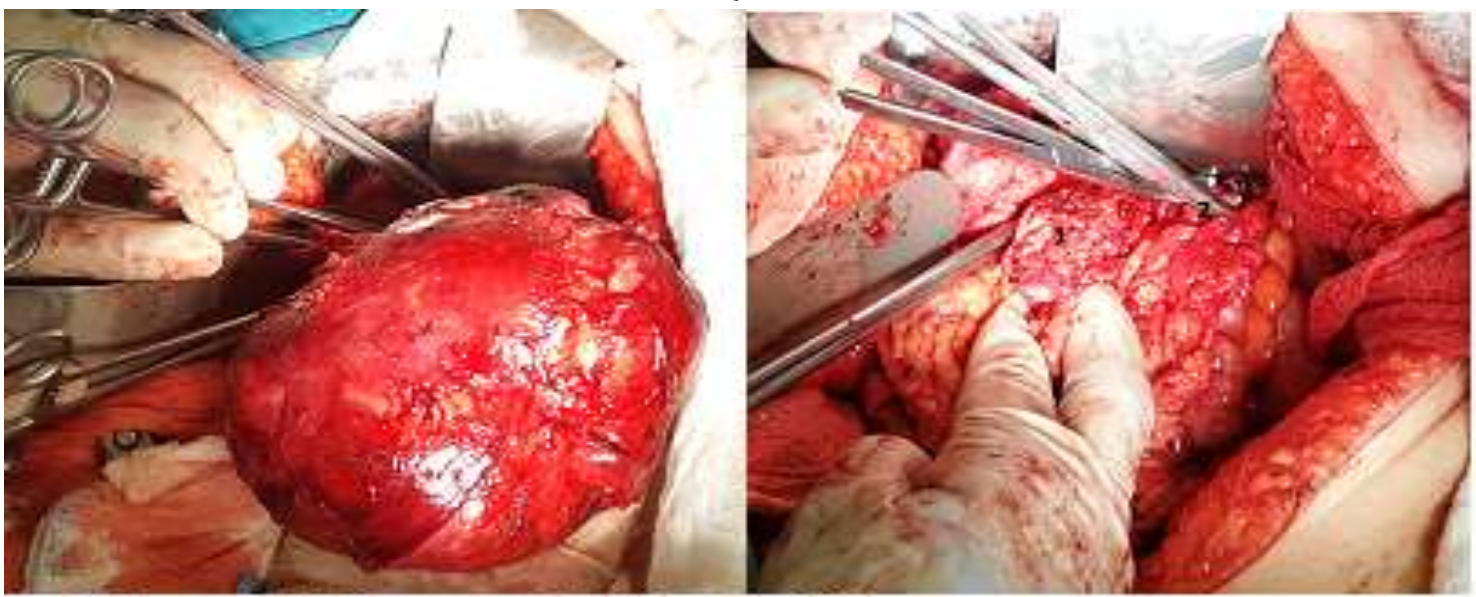

\section{Cabeza del Páncreas; 2. Hilio Esplénico}

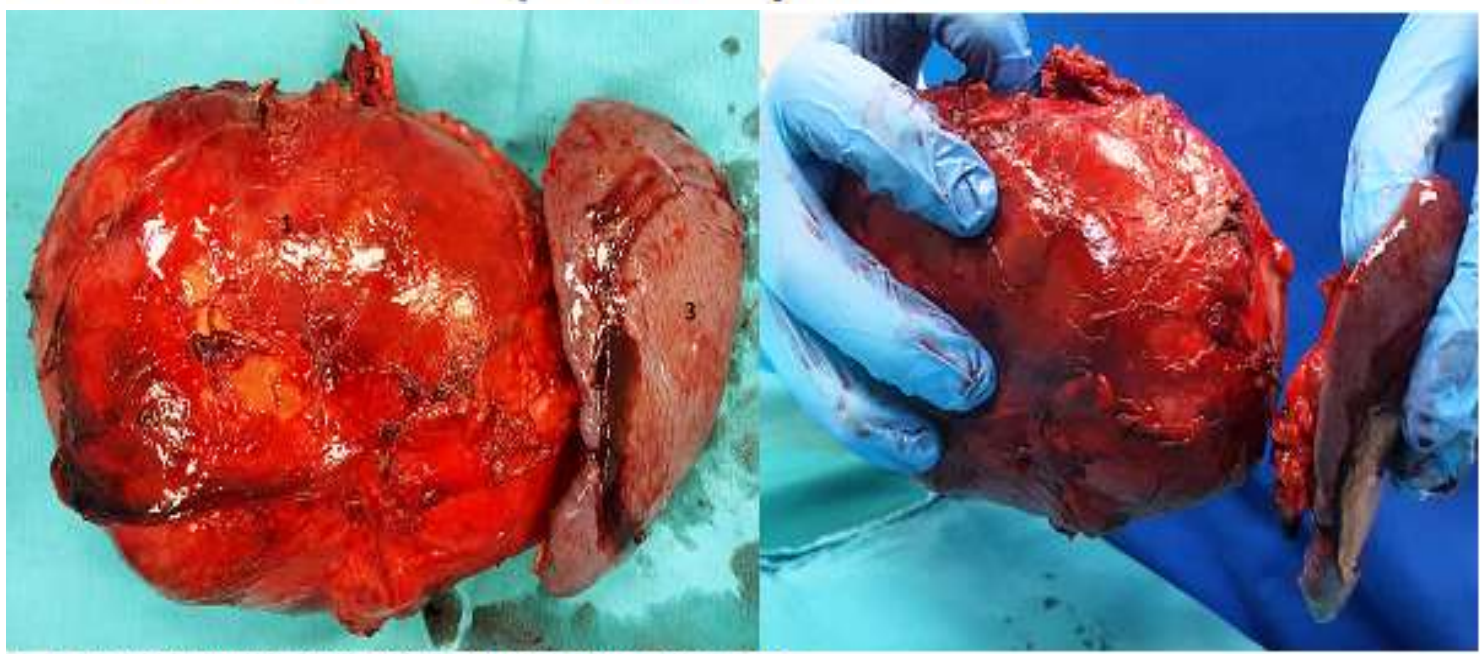

1. Pancreatectomía distal; 2. Hilio Esplénico; 3. Bazo

Fuente: Procedimiento Quirúrgico Solca Chimborazo 2018 
Histopatológico: Tumor consistente del saco vitelino extra gonadal de ubicación peri pancreática (capsula integra, bazo negativo para infiltración tumoral, congestión vascular, hilio de bazo se aíslan tres ganglios negativos para malignidad)

Gráfico No5. Histopatologico

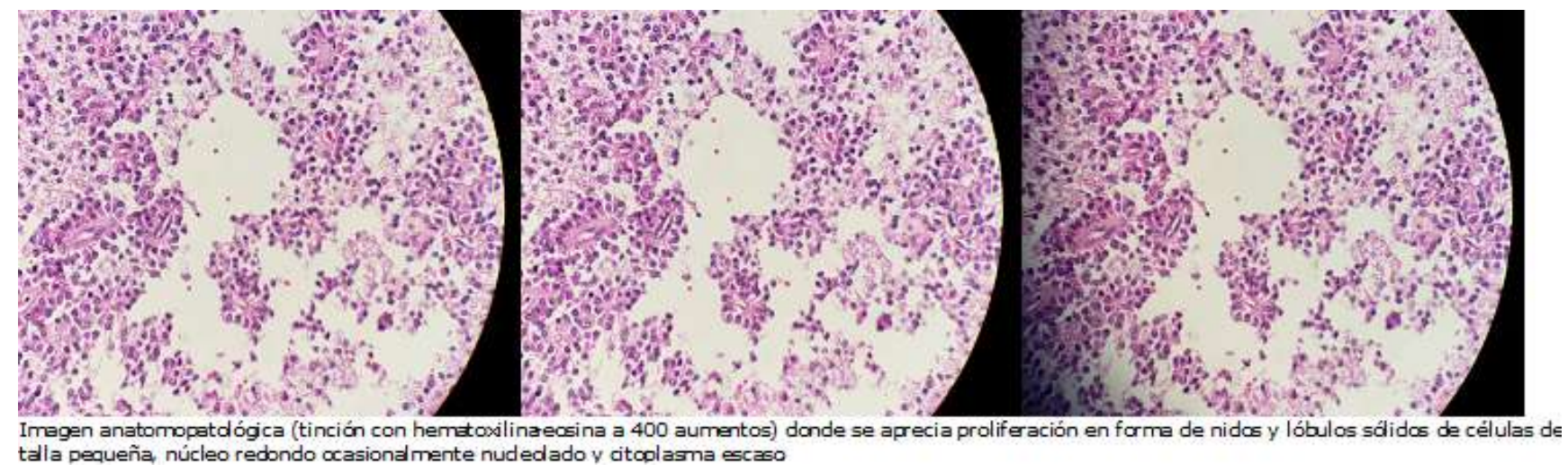

Fuente: Servicio De Patologia Solca Chimborazo2018

Inmunohistoquimica: Vimentina: +++; CD56(MRQ42): +++; Alfafetoproteina: ++; Ki67: Negativo; Cromogranina A: negativa; Queratina AE1/AE3: Negativo; IDG: Tumor Solido Pseudopapilar de Pancreas (FRANTZ GRUBE)

Gráfico No6 Inmunohistoquimica:

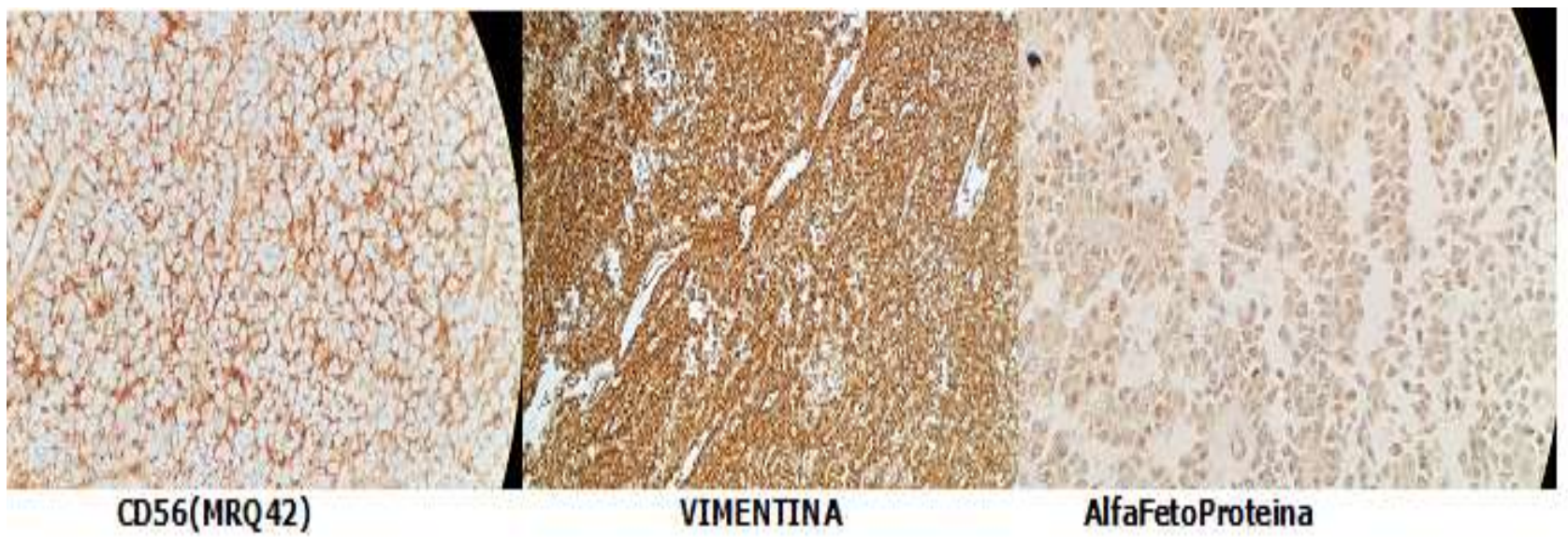

Fuente: Servicio de Patología Solca Chimborazo 2018

\section{Discusión.}

El tumor pseudopapilar sólido (SPT) del páncreas es el tumor menos frecuente de localización pancreática en menos de $1 \%$, se encuentra en mayor proporción en mujeres de edad menor a los 20 años, se clasifica como un carcinoma de bajo potencial de malignidad 
El diagnóstico preoperatorio se realiza mediante la TC y la RM. En la TC con contraste, donde aparece una masa delimitada de densidad heterogénea por la presencia de áreas hipo densa en cavidades quísticas necrótico-hemorrágicas. Se asientan en cuerpo-cola del páncreas, presentan formas multicéntricas, en este caso los datos más característicos de la RM son la presencia de una tumoración bien delimitada con áreas sólidas izo intensas con el páncreas en T1 o bien una señal ligeramente alta en T2.

En la práctica, ante una lesión potencialmente maligna y en ausencia de antecedentes de pancreatitis aguda, la prudencia recomienda considerarla y tratarla como una neoplasia quística, sin retrasar la intervención quirúrgica.

El tratamiento de elección es la cirugía, ya que la mayoría de los TPSP son potencialmente curables por resección quirúrgica, que deberá ser lo más extensa posible. Presenta escasa mortalidad, bajas tasas de recidiva y metástasis, sobre todo, una buena supervivencia a largo plazo. En ocasiones el tumor alcanza un gran tamaño, comprometiendo las estructuras vecinas y haciendo imposible su resección o a sus ves requiriendo la extracción de órganos vecinos, pero aún en estos casos hay una larga sobrevida.

\section{Conclusiones}

- Es un tumor poco frecuente con bajo grado de malignidad sin embargo su curación es mediante resección quirúrgica.

- Las manifestaciones clínicas pueden confundir su diagnóstico, la sospecha clínica en los grupos etarios de mayor prevalencia es clave para su manejo.

- La confirmación del diagnóstico se lo realizara bajo inmunohistoquimica le histopatológico de la muestra luego de la resección quirúrgica sin embargo las manifestaciones clínicas y exámenes de imagen son necesarias para su identificación.

\section{Referencias Bibliográficas}

1. Tumor de Frantz: Neoplasia Sólida Pseudopapilar de Páncreas, Juan Llatas, Américo Palomino y Oscar Frisancho, Rev. Gastroenterol. Perú; 2011; 31-1: 56-60.

2. Tumor de Frantz reporte de casos, Andrea Tróchez R., M.D. , Rafael R. Peña, M.D., Cirujano Pediatra y Martín Gómez T. M.D., Cirujano Pediatra, Revista Fac Med. Volumen 17 - No. 2 - Julio - Diciembre de 2009.

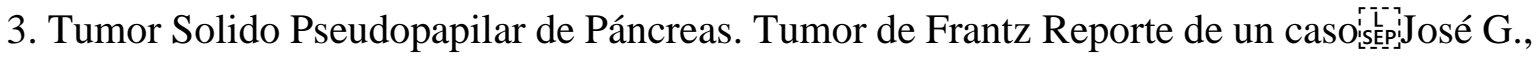
López Añez; Verónica, González- Vilar ; Mónica, Duque Yonekura ; Rafael A., GonzálezDuque; Ana V., Vargas-Gonzáles.

4. Solid and Cystic Pseudopapillary Tumor of the Pancreas: A Case Report Bojan Z. Milošević, Rade M. Marković, Aleksandar M. Cvetkovic Srp Arh Celok Lek. 2013 MayJun;141(5-6):384-386

5. Tumor de Frantz. Presentación de un caso Frantz's tumor. Report of a case Drs. Rafael Cerdan P., Jose Ignacio Barranco D., Sonia Cantin B., Joaquin Bernal J., Alejandro Serrano R.1, Jesús María Esarte M. Rev. Chilena de Cirugía. Vol 59 - No 2, Abril 2007.

6. Original Report MR Imaging Features of Solid Pseudopapillary Tumor of the Pancreas 
in Adult and Pediatric Patients, Vito Cantisani Koenraad, J. Mortele Angela Levy Jonathan N. Glickman Paolo Riccis Roberto Passariello Pablo R. Rosisepistuart G. Silverman AJR:181, August 2003.

7. Solid Pseudopapillary Tumor of the Pancreas with Massive Cystic Degeneration; Source of Diagnostic Confusion Austin J Ostermeier and Michael B Nicholl and Ostermeier, Pancreat Disord Ther 2014, 4:3 DOI: 10.4172/2165-7092.1000145

8. Tumor de Gruber Frantz. Presentación de caso clínico y revisión de literatura Dr. García C, César, Dra. Lili Naranjo M, Dra. Verónica Cajas C, Dra. Jenny Calle G, Dra. Patricia Bermeo S. Dr. Darío Cullquicondor S. Medico Tratante del Servicio de Cirugía Oncológica del Hospital Naval Guayaquil.

9. Solid Pseudopapillary Epithelial Neoplasm - a rare but curable pancreatic tumour in young women M. FROST, M.D. Departments of Surgery, University of Cape Town and Oregon Health and Science University, Portland, Oregon, USA, VOL 49, NO. 2, APRIL 2011 SAJS.

10. Tumor de Frantz-Gruber: Revisión de la bibliografía a propósito de un caso. FrantzGruber Tumor: revisión en relación a un caso, Autores: Dr Ricardo Arnaldo Torres Ávila, Dra. Jianeya Manzano Suárez Dr Goar V González Álvarez, Dra Jasmine Josette Ellis-Davy Dr Alejandro E Armesto Pérez, Dra Romey Camue Luis .

11. Clinicopathologic characteristics and surgical treatment of solid pseudopapillary tumor of the pancreas: Revisión de la bibliografía a propósito de un caso, Autor: Huang Y1, Feng JF2, ; Nursing Department, Zhejiang Cancer Hospital, Hangzhou, China; Department of Oncological Surgery, Zhejiang Cancer Hospital, Hangzhou, China

12. Tumor sólido pseudopapilar de páncreas. Presentación de un caso y revisión de la literatura Autor: José Francisco Camacho-Aguilera, César Romero-Mejía, Alfonso Valenzuela Espinoza; www.medigraphic.org.mx 


\section{PARA CITAR EL ARTÍCULO INDEXADO.}

Haro Erazo, C., \& Salinas Mancheno, F. (2019). Tumor de Frantz : Presentación de un caso clínico. Ciencia Digital, 3(3), 488-496. https://doi.org/10.33262/cienciadigital.v3i3.664

\section{Ciencia \\ LDigital}

El artículo que se publica es de exclusiva responsabilidad de los autores y no necesariamente reflejan el pensamiento de la Revista Ciencia Digital.

EI artículo queda en propiedad de la revista y, por tanto, su publicación parcial y/o total en otro medio tiene que ser autorizado por el director de la Revista Ciencia Digital.
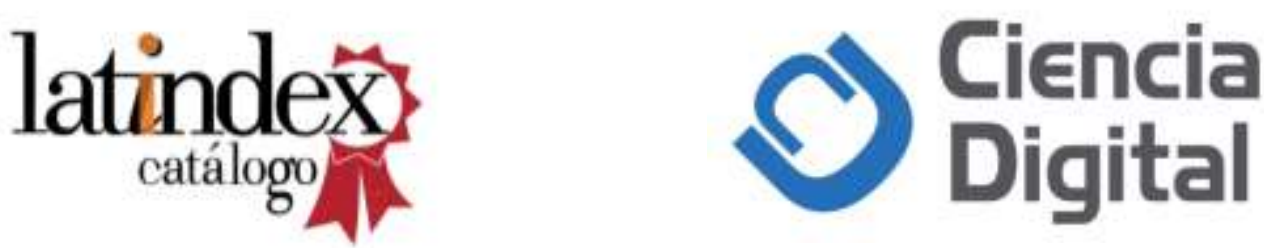